\title{
The Bermuda Triangle of d- and f-MRI sailors - software for susceptibility distortions (SDCFlows)
}

Esteban, Oscar ${ }^{1}$; Adebimpe, Azeez ${ }^{2}$; Markiewicz, Christopher J. ${ }^{3}$; Goncalves, Mathias ${ }^{3}$; Blair, Ross W. ${ }^{3}$; Cieslak, Matthew ${ }^{2}$; Naveau, Mikaël ${ }^{4}$; Sitek, Kevin R. ${ }^{5}$; Sneve, Markus H. ${ }^{6}$; Provins, Céline ${ }^{1}$; MacNicol, Eilidh ${ }^{7}$; Satterthwaite, Theodore D. ${ }^{*}$;

Poldrack, Russell A..$^{*}$

\footnotetext{
${ }^{1}$ Dep. of Radiology, Lausanne University Hospital and University of Lausanne; ${ }^{2}$ Department of Psychology, Stanford University; ${ }^{3}$ Perelman School of Medicine, University of Pennsylvania, PA, USA; ${ }^{4}$ Cyceron, UMS 3408 (CNRS -

UCBN), France; ${ }^{5}$ Dept. of Communications Science and Disorders, University of Pittsburgh; ${ }^{6}$ Center for Lifespan Changes in Brain and Cognition, University of Oslo; ${ }^{7}$ Dept. of Neuroimaging, Institute of Psychiatry, Psychology and Neuroscience, King's College London, London, UK.

${ }^{*}$ Authors contributed equally
}

Introduction: Echo-Planar Imaging (EPI) allows very fast acquisition of whole-brain data, which enables standard functional \& diffusion MRI (f/dMRI). However, EPI is notably sensitive to variations in the base $B_{0}$ field. Small deviations in parts-per-million from the nominal $B_{0}$ caused by steps in magnetic susceptibility (tissue interfaces) introduce misplacements in the registered location of voxels of up to some $\mathrm{cm}$ in standard settings along the phase-encoding direction (PE), apparent as local geometrical distortions of the imaged specimen. In humans, the susceptibility distortion (SD) is prominent starting at the petrous bone and extending towards the ear canals, defining a sort of triangle where signal vanishes (Fig. 1). SD is well-known, but existing solutions require mapping $B_{0}$ deviations and are sensitive to several imaging parameters. In practice, addressing SDs is error-prone and often overlooked. Here, we introduce SDCFlows (SD Correction Flows), an open-source utility that leverages BIDS ${ }^{1}$ and several existing software tools to provide standardized, best-effort SD correction.

Methods: SDCFlows defines a clear application programming interface (API) that divides the problem into 2 stages:

1) Estimation - the MRI acquisitions in the protocol for $S D$ are discovered and preprocessed to estimate a map of $B_{0}$ non-uniformity in $\mathrm{Hz}\left(\Delta \mathrm{B}_{0}\right)$. The theory behind these distortions is well described in the literature ${ }^{3,4}$ (Fig. 1). SDCFlows builds on freely-available software to implement three major strategies for estimating $\Delta \mathrm{B}_{0}$ (Eq. 1). The first strategy uses spiral-echo imaging, which directly produces $\Delta \mathrm{B}_{0}$ maps, or other schemes such as gradient-recalled echo imaging that measure the voxel-wise phase drift (proportional to $\Delta \mathrm{B}_{0}$ ) between two subsequent echoes. These techniques stem from the analytical solution to the distortion (Eq. 1), and SDCFlows refines the widespread workflow based on FSL (PRELUDE+FUGUE). The second strategy uses methods exploiting the symmetry of the distortion when the PE polarity is reversed, offering two implementations based on FSL TOPUP ${ }^{6}$ and AFNI 3dQwarp ${ }^{7}$. Third, SDCFlows integrates fMRIPrep's "fieldmap-less" technique ${ }^{8}$ that nonlinearly registers the EPI scan to the same subject's anatomical MRI.

2) Application - the estimated $\Delta \mathrm{B}_{0}$ map is aligned to the target EPI scan to be corrected and used to generate a displacement field in NIfTI format that is compatible with $\mathrm{ANTs}^{2}$. The voxel location error along the PE will be proportional to $\Delta \mathrm{B}_{0} \mathrm{~T}_{\text {ro }}$ (Fig. 1).

\section{Results:}

A standard software tool that democratizes the implementation of an otherwise elusive problem. We have refactored SDCFlows from fMRIPrep's codebase and built an effective API for reuse in any workflow for preprocessing EPI images. The API is split into 1) estimation and 2) correction, so that workflow developers may combine the effects of SD with other spatial corrections of the image and implement "1-step" resamplings. The API minimizes computational duplication; e.g., fMRIPrep runs estimation for each target image to be corrected.

A thoroughly tested tool that leverages existing implementations. SDCFlows implements workflows leveraging existing tools and supports them to cover a wide range of protocols. We are pushing the BIDS specifications to better capture fieldmaps ${ }^{9}$. All estimation strategies are regularized through B-Spline approximation, which makes the outputs consistent across strategies and opens new avenues for a systematic comparison of corrections. 
A thoroughly documented and self-reporting tool. SDCFlows follows "glass-box" principles ${ }^{8}$ and produces "reportlets" to visually assess the results (Fig. 2).

Conclusion: SDCFlows documents the susceptibility distortion artifact and equips $\mathrm{d} / \mathrm{fMRI}$ researchers with a reliable and transparent tool to address the problem.

\section{References:}

1. Gorgolewski, K. J. et al. The brain imaging data structure, a format for organizing and describing outputs of neuroimaging experiments. Sci. Data 3, 160044 (2016).

2. Avants, B. B. et al. A reproducible evaluation of ANTs similarity metric performance in brain image registration. NeuroImage 54, 2033-44 (2011).

3. Jezzard, P. \& Balaban, R. S. Correction for geometric distortion in echo planar images from B0 field variations. Magn. Reson. Med. 34, 65-73 (1995).

4. Hutton, C. et al. Image Distortion Correction in fMRI: A Quantitative Evaluation. NeuroImage 16, 217-240 (2002).

5. Jenkinson, M. Fast, automated, N-dimensional phase-unwrapping algorithm. Magn. Reson. Med. 49, 193-197 (2003).

6. Andersson, J. L. R., Skare, S. \& Ashburner, J. How to correct susceptibility distortions in spin-echo echo-planar images: application to diffusion tensor imaging. NeuroImage 20, 870-888 (2003).

7. Cox, R. W. \& Hyde, J. S. Software tools for analysis and visualization of fMRI data. NMR Biomed. 10, 171-178 (1997).

8. Esteban, O. et al. fMRIPrep: a robust preprocessing pipeline for functional MRI. Nat. Methods 16, 111-116 (2019).

9. Esteban, O. BIDS Specification proposed changes (\#622): Allow encoding the fieldmapping intent of the protocol. https://github.com/bids-standard/bids-specification/pull/622. 


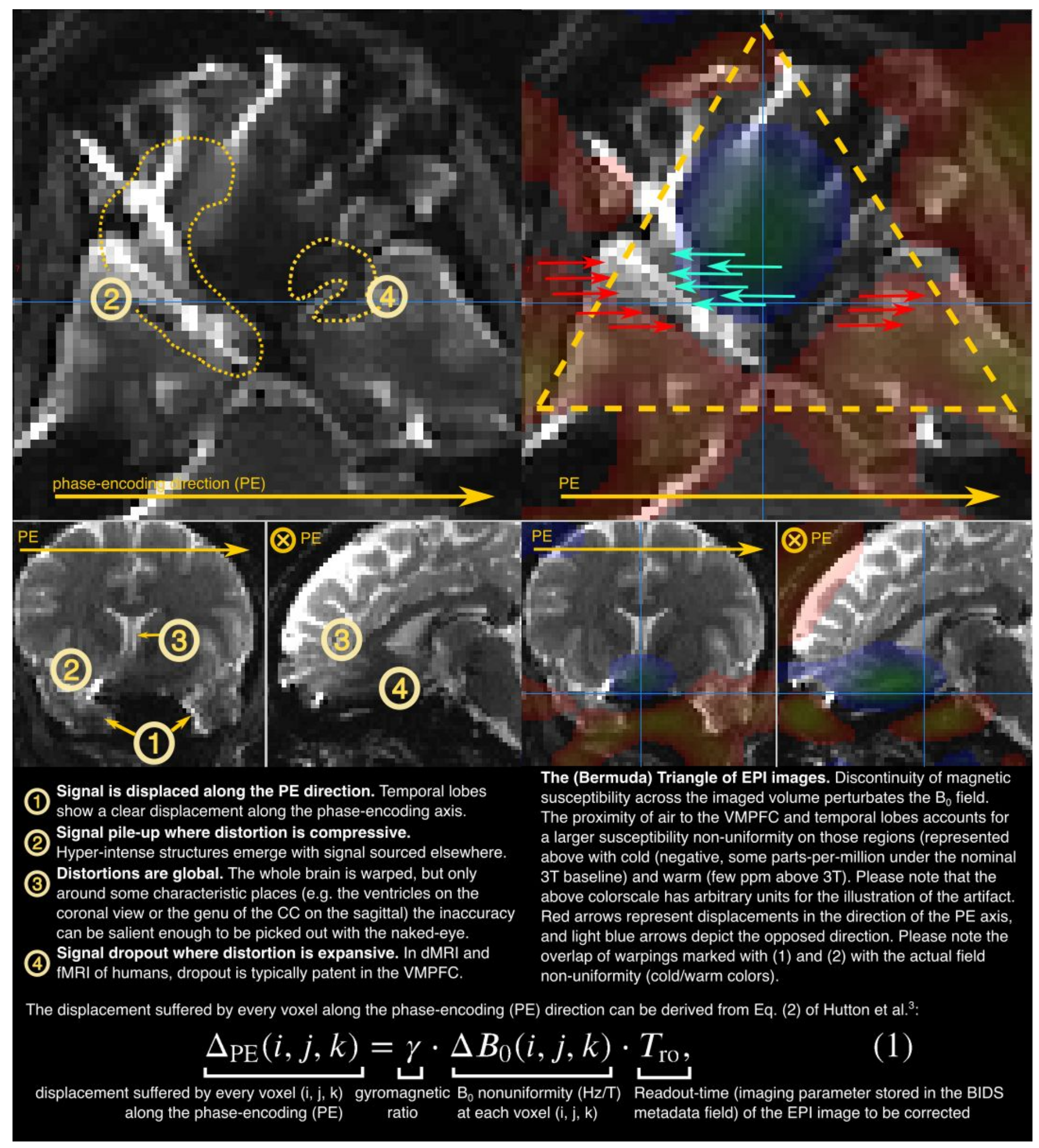

Figure 1. The problem of susceptibility distortions. The annotated panels show one EPI image from one subject (with identifier 101006) of the Human Connectome Project. The views on the right-hand side also have an overlay of the field map estimated with the PEPOLAR (PE-Polarity) technique based on FSL TOPUP. 


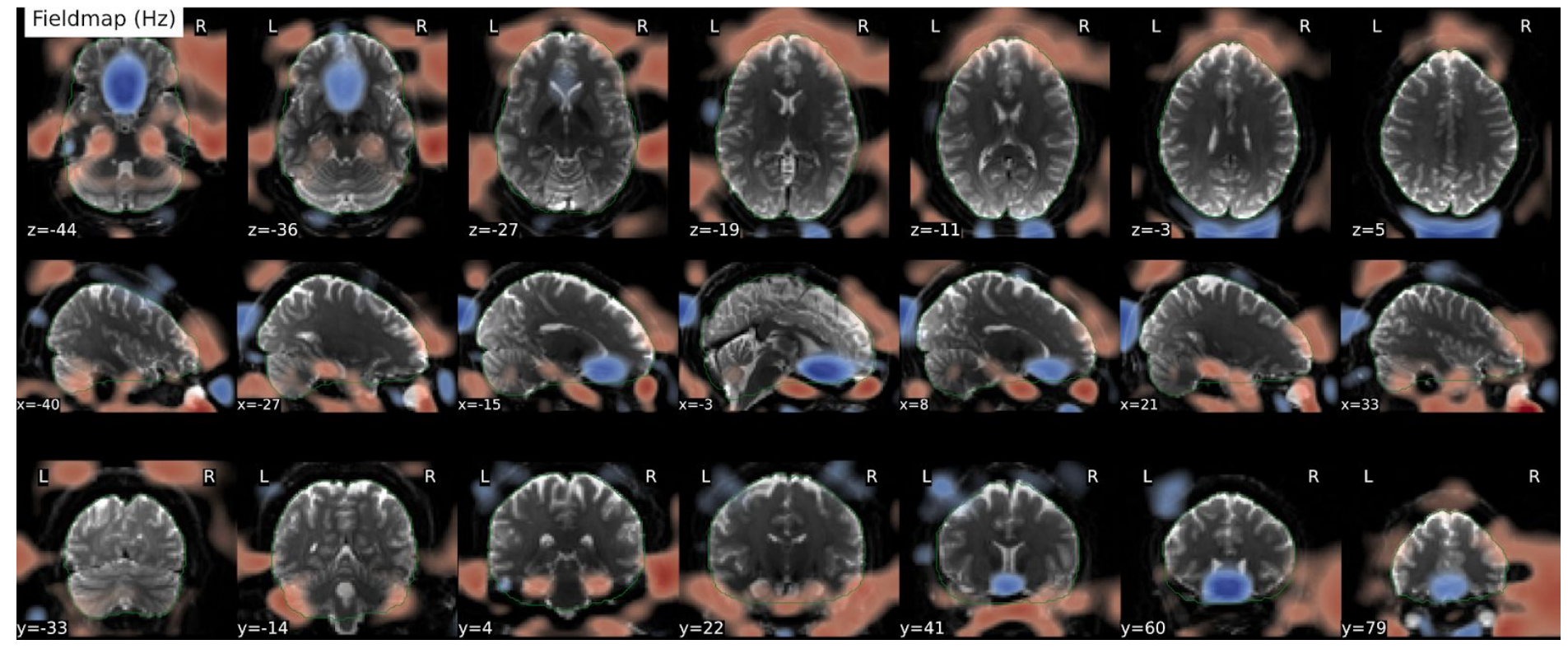

Figure 2. SDCFlows produces self-contained, dynamic visualizations of the estimated $\Delta \mathrm{B}_{0}$ maps in scalable-vector graphics (SVG) format. These "reportlets" (or "mini-reports") are easily embedded in HTML visual reports, such as those produced by $f M R I P r e p$ and $d M R I P r e p$. 